\title{
OVERMODED HIGH-POWER RF MAGNETIC SWITCHES AND CIRCULATORS*
}

\author{
Sami Tantawi**, SLAC, Menlo Park, CA 94025, USA
}

\begin{abstract}
We present design methodology for active rf magnetic components which are suitable for pulse compression systems of future X-band linear colliders. These components comprise an array of active elements arranged together so that the total electromagnetic field is reduced and the power handling capabilities are increased. The active element of choice is a magnetic material (garnet), which can be switched by changing a biasing magnetic field. A novel design allows these components to operate in the low loss circular waveguide mode $\mathrm{TE}_{01}$. We describe the design methodology, the switching elements and circuits.
\end{abstract}

\section{INTRODUCTION}

Because of the requirements of the Next Linear Collider high-power rf systems, passive microwave components have developed considerably during the last few years $[1,2]$. The power handling capabilities of these components have increased considerably [3]. This has been achieved by increasing the size of these components with respect to the operating wavelength, i.e., by overmoding these components. Similar attempts have been made to increase the power handling capabilities of active semiconductor components. A switch made from a PIN diode array has been developed to operate in an overmoded waveguide carrying the $\mathrm{TE}_{01}$ mode [4]. Optically controlled semiconductor switches operating in overmoded waveguides have also been reported [5].

In this spirit we suggest geometries that allow nonreciprocal devices to operate in an overmoded waveguide structures. We present the theory for a nonreciprocal device that operates in the coaxial circular waveguide mode $\mathrm{TE}_{01}$. This device has the potential of handling tens of megawatts at X-band. It could be used as a circulator or as a switch.

\section{DEVICE GEOMETRY}

The implementation of a circulator or a switch can be achieved using a two-port nonreciprocal network plus a $3 \mathrm{~dB}$ hybrid and a splitter, see Fig. 1. The simplest implementation of the nonreciprocal element in an overmoded waveguide using the $\mathrm{TE}_{01}$ mode is shown in Fig. 2. This implementation depends on the so-called

*Work supported by the U>S. Department of Energy contract DEAC03-76SF00515

**Also with the communications and electronic Department, Cairo University, Giza, Egypt. wrap-around mode converter [3]. In this system a mode converter launches the $\mathrm{TE}_{01}$ mode, which has both axial and radial magnetic fields. At the end of the mode converter the coaxial guide becomes narrow and only the

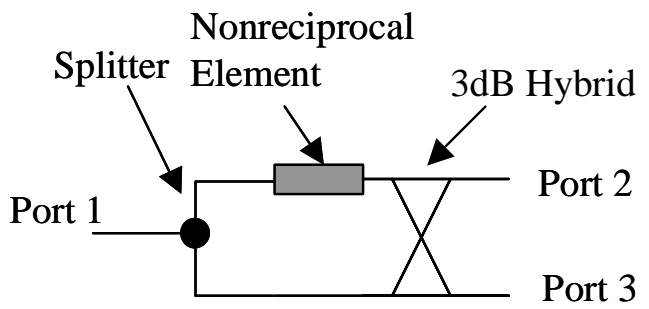

Figure 1. The three-port network will work as a circulator if the phase shift through the two-port nonreciprocal element is different by 180 degrees for different propagation direction. The system would work as a switch if one can control the phase shift through the element.

coaxial TEM mode can propagate, see Fig. 2. A pulsed current signal could be launched from that narrow port (port-A in Fig. 2) This pulse would have only azimuthal magnetice field. This field is used to bias a piece of garnet wrapped around the center conductor of the coaxial structure. This structure has several advantges for handling high power rf signals:

1- Since we are operating with an overmoded waveguide that has a larger cross sectional area for a given wavelength, the device should be able to handle more power.

2- All rf electric field lines are parallel to the interface between the garnet material and vacuum; see the theory section below.

3- The center conductor could be used to cool the garnet material. It could be made as a tube with water flowing in the middle.

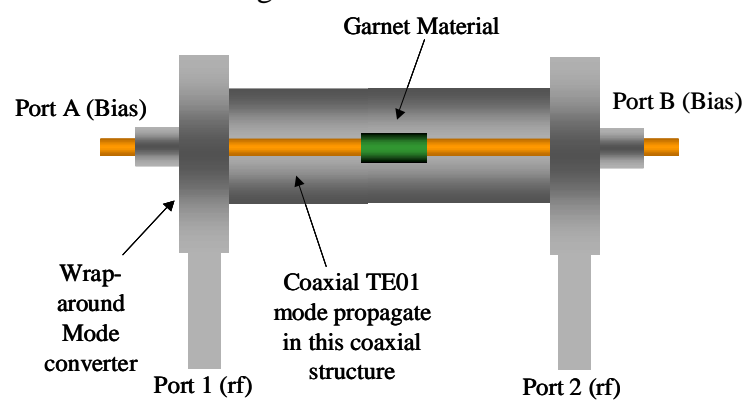

Figure 2. Nonreciprocal two-port device employing the $\mathrm{TE}_{01}$ mode.

Several variations on this geometry are possible. To implement the system shown in Fig. 1 elegantly we 
suggest the geometry shown in Fig. 3. In this geometry the splitter is realized by dividing the power between the $\mathrm{TE}_{01}$ and the $\mathrm{TE}_{02}$ modes. These two modes interact with the garnet coated section in different manners. To implement the circulator, one could design the system to make the phase difference between these two modes in the forward direction differ from that of the backward direction by $\pi$. To implement a switch, one can control that phase shift by varying the current in the center conductor.

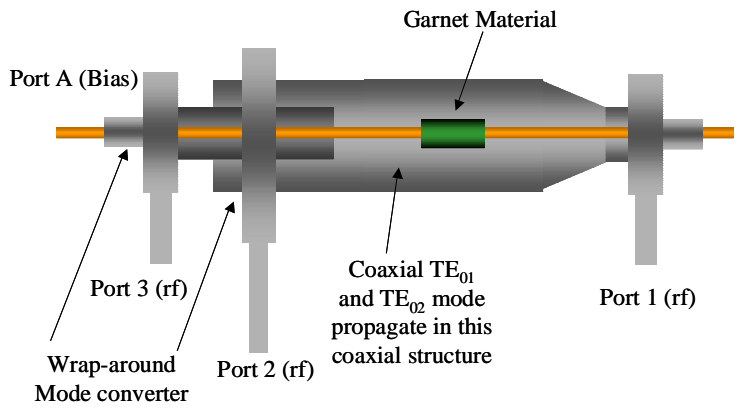

Figure 3. Implementation of the overmoded circulator/switch.

In the reminder of this paper we will analyze and present a theory for propagating modes with no azimuthal variation in the coaxial structure coated with a layer of garnet.

\section{THEORY}

Consider the waveguide structure shown in Fig. 4. We assume a DC current in the center conductor. Using cylindrical coordinates, the magnetic permeability tensor of the garnet material is given by:

$$
\mu_{g}=\mu_{0}\left(\begin{array}{ccc}
\mu & 0 & -j k \\
0 & 1 & 0 \\
j k & 0 & \mu
\end{array}\right)
$$

Here, $\mu_{0}$ is the free space permeability and $\mu$ and $k$ are complex parameters that depend on the biasing current. For a detailed derivations of this tensor see, for example, [6]. Consider a solution for the time harmonic Maxwell's equations in which there is no variation with the azimuth; i.e. $\frac{\partial}{\partial \phi}=0$, and $E_{z}=0$; i.e. a TE mode.

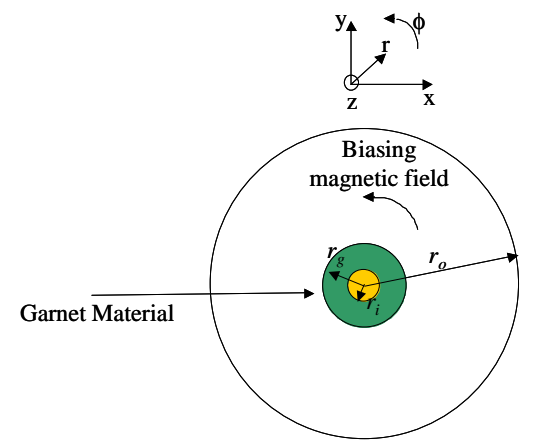

Figure 4. Geometry of the overmoded waveguide.

These two conditions immediately imply that $H_{\phi}=0$, and even, when applying Eq. (1) it follows that $E_{r}=0$. Maxwell's equations inside the garnet region reduce to the following set of equations:

$$
\begin{aligned}
& \frac{\partial H_{z}}{\partial r}=-j\left(\omega \varepsilon E_{\phi}+\beta H_{r}\right), \\
& \frac{\beta}{\omega \mu_{0}} E_{\phi}=j k H_{z}-\mu H_{r}, \\
& \frac{1}{\omega \mu_{0}}\left(\frac{E_{\phi}}{r}+\frac{\partial E_{\phi}}{\partial r}\right)=k H_{r}-j \mu H_{z} ;
\end{aligned}
$$

where $\omega$ and $\beta$ are the angular frequency and propagation constant respectively, and $\varepsilon$ is the permitivity of the garnet material. Solving Eq. (2) and (3) for $H_{r}$ and $E_{\phi}$ and substituting in (4) one get the following second order deferential equation for $H_{z}$,

$$
r \frac{\partial^{2} H_{z}}{\partial r^{2}}+\frac{\partial H_{z}}{\partial r}-\left(q \beta+r \gamma^{2}\right) H_{z}=0 ;
$$

where $\gamma \equiv \sqrt{\beta^{2}+k_{g}^{2}\left(k^{2}-\mu^{2}\right) / \mu}, k_{g}^{2} \equiv \omega^{2} \mu_{0} \varepsilon$, and $q \equiv k / \mu$. Here we make the simplifying assumption that the permeability tensor is independent of radius. Solving Eq. (5) while using Eqs. (2) and (3) yield an expression for the radial impedance,

$$
Z_{g} \equiv \frac{E_{\phi}}{H_{z}}=\mu \frac{j \omega \mu_{0}}{\left(\beta^{2}-k_{0}^{2} \mu\right) / \gamma}\left(\delta-\frac{2\left(C M^{\prime}(\delta / 2,1,2 r \gamma)+U^{\prime}(\delta / 2,1,2 r \gamma)\right.}{C M(\delta / 2,1,2 r \gamma)+U(\delta / 2,1,2 r \gamma)}\right)
$$

where $U(., .,$.$) and M(., .,$.$) are the confluent$ hypergeometric functions, ()$^{\prime}$ means a derivative with respect to the third argument, $\delta \equiv 1+q \beta / \gamma$, and $C$ is a constant. Eq. (6) becomes valid also for the vacuum region if one lets $\mu \rightarrow 1$ and $k \rightarrow 0$. The resultant simplified equation is

$$
Z_{0}=j \frac{\omega \mu_{0}}{\gamma_{0}} \frac{K_{1}\left(\gamma_{0} r\right)-\bar{C} I_{1}\left(\gamma_{0} r\right)}{K_{0}\left(\gamma_{0} r\right)+\bar{C} I_{0}\left(\gamma_{0} r\right)}
$$


where $K$ and $I$ are the modified Bessel functions, $\gamma_{0} \equiv \sqrt{\beta^{2}-k_{0}^{2}}, k_{0}^{2} \equiv \omega^{2} \mu_{0} \varepsilon_{0}, \quad \varepsilon_{0}$ is the free space permittivity, and $\bar{C}$ is a constant.

The boundary conditions of the problem can now be applied by setting both $Z_{g}$ and $Z_{0}$ equal to zero at the inner and outer radii respectively. This would yield the two Constants $C$ and $\bar{C}$. Now, equating $Z_{g}$ and $Z_{0}$ at the interface between the two media, at $r=r_{g}$, yield the required equation for the propagation constant $\beta$.

\section{RESULTS}

For a proof of principle numerical experiment we considered the properties of calcium vanadium doped garnet [7]. We choose this material because of its narrow line width. The calculated values for $\mu$ and $k$ are shown in Fig. 5 and 6. The operating frequency used to generate these curves is $11.424 \mathrm{GHz}$. The dielectric constant of this material is about 14 .

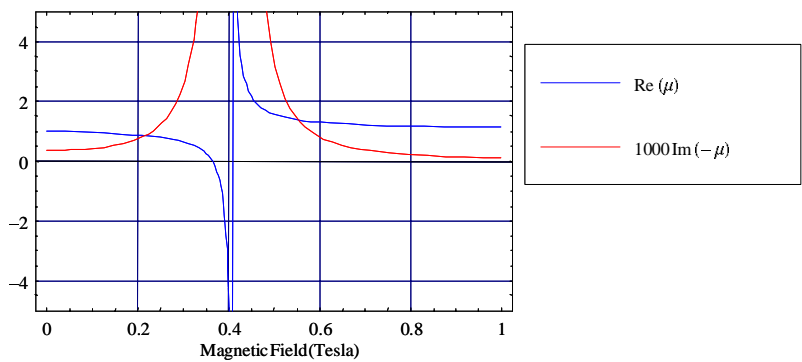

Figure 5. Variations of the diagonal elements of the relative magnetic permeability tensor, $\mu$, with magnetic field.

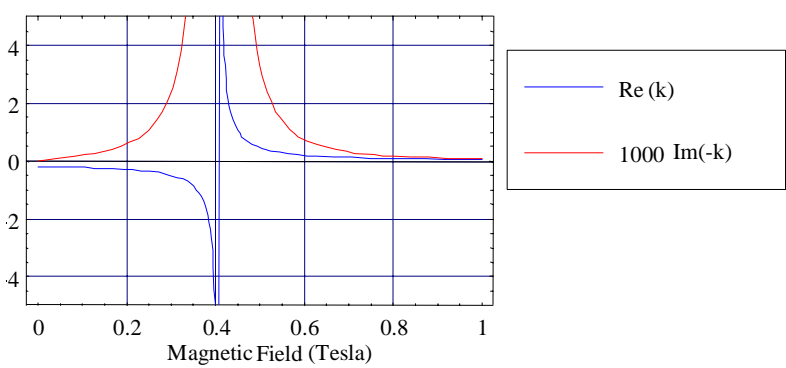

Figure 6 Variations of the off diagonal elements of the relative magnetic permeability tensor, $k$, with magnetic field.

The biasing magnetic field was chosen so that the material would operate below the resonance frequency.

Figure 7. Shows the required garnet thickness and length for a $\pi$ phase difference between forward and backward $\mathrm{TE}_{01}$ mode waves. For the optimum thickness, the rf losses are less than one percent. This is a manageable losses level, which be cooled through the center conductor.
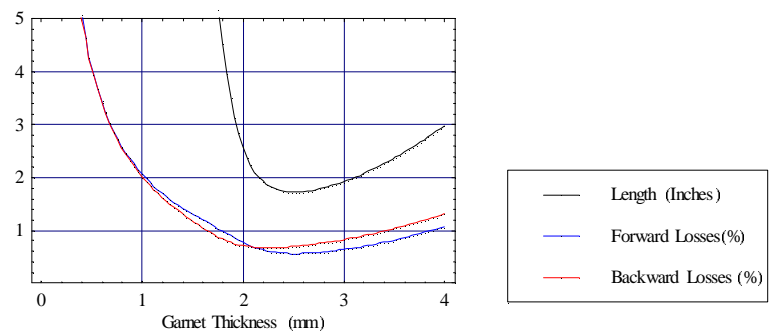

Figure 7. The required dimensions of the garnet material for a $\pi$ phase shift between forward and backward waves for the $\mathrm{TE}_{01}$.

\section{CONCLUSION}

We presented a novel design for circulators and switches in an overmoded waveguide. These designs increase the power handling capabilities of these devices. We also presented a theory for the design and estimated the dimensions of the nonreciprocal material and rf losses for these devices.

\section{ACKNOWLEDGMENT}

The author would like to thank R. Ruth, V. Dolagashev, P. Wilson, N. Kroll, R. Miller, F. Tamura for many useful discussions.

\section{REFERENCES}

[1]Sami G. Tantawi, "New Development in rf Pulse Compression," Sami G. Tantawi, Proc. Of the International XX-Linac Conference, Monterey, California, USA, August 21 - 25, 2000, p. 673-677

[2]C. Nantista and S. Tantawi "Multi-Moded Passive RF Pulse Compression Development at SLAC, " to be pulblished in the Proc. of Advanced Accelerator Concepts Workshop, Santa Fe, NM, June 10-16, 2000. AIP Proceedings 569. Edited by Patrick L. Colestock.

[3]Sami G. Tantawi, et. al. "The Generation Of 400-MW RF Pulses At X Band Using Resonant Delay Lines,", IEEE Trans. on Microwave Theory and Techniques, Vol 47, No. 12, December, 1999, p. 2539-2546

[4]Fumihiko Tamura, Sami G. Tantawi, "Development Of High Power X-Band Semiconductor Rf Switch For Pulse Compression Systems Of Future Linear Colliders," Proc. of the 20th International Linac Conference (Linac 2000), Monterey, California, 21-25 Aug 2000, vol. 2, pp 751-753.

[5]Sami G. Tantawi, Ronald D. Ruth, Arnold E. Vlieks, and Max Zolotorev, "Active High-Power RF Pulse Compression Using Optically Switched Resonant Delay Lines," IEEE Transaction on Microwave Theory and Techniques, Vol. 45, No 8, August 1997, p. 14861492.

[6]R. E. Collin, "Foundations for Microwave Engineering,” McGraw-Hill, Inc., 1966, p. 294.

[7]http://www.trans-techinc.com/catalog/pdfs/pg1 4\&5.pdf. 